\title{
Ipsilateral limbal translocation for treatment of partial limbal deficiency secondary to ocular alkali burn
}

\author{
M Cristina Nishiwaki-Dantas, Paulo Elias Correa Dantas, José Ricardo A Reggi
}

\begin{abstract}
Backgroundlaim-Chemical ocular burn, especially by alkali, may result in damage to the limbal stem cells, fundamental in maintaining the integrity of ocular surface. Clinically, patients manifest abnormal conjunctival-corneal healing with peripheral neovessels, chronic epithelial defect, severe stromal inflammation, and conjunctivalisation of the corneal, which is characteristic of limbal deficiency. Penetrating keratoplasty has a poor prognosis in these cases. Available therapeutic options included limbal autograft and allograft transplantation associated or not with human amniotic membrane transplant. The authors propose an alternative treatment with ipsilateral autologous limbal transplantation, transferring the healthy portion of the limbus from the partially burned eye to the deficient area in patients with partial limbal deficiency secondary to alkali burn.
\end{abstract}

Methods-Ipsilateral limbal autografts were performed in five patients with partial limbal deficiency secondary to alkali burn, transferring the healthy portion of the limbus to the burned area of the same eye, without intervening in the contralateral eye.

Results-All cases had regression of the limbal deficiency with improvement of visual acuity to a minimum of 20/60-20/20 (partial), without complications during the follow up period (range 7-11 months). Conclusion-Ipsilateral autologous limbal autograft is a therapeutic option for partial limbal deficient eyes.

(Br f Ophthalmol 2001;85:1031-1033)

Limbal stem cells are considered to be located at the base of limbal epithelium and are responsible for the repopulation of corneal epithelium. They also act as a junctional barrier, not allowing conjunctival tissue to grow over the cornea. Its dysfunction or limbal deficiency could lead to a sequence of abnormal phenomena on the ocular surface characterised by abnormal corneoconjunctival healing, corneal neovascularisation, chronic epithelial defects, severe stromal inflammation, and conjunctivalisation leading to corneal opacification and loss of visual acuity. ${ }^{12}$ Limbal deficiency could be caused by ocular burn (alkali or acid), ocular cicatricial diseases (Stevens-Johnson syndrome, ocular cicatricial pemphygoid), and keratopathy induced by contact lens wear. ${ }^{3-7}$

There are several strategies to manage limbal deficiency. In 1977, Thoft $^{8}$ proposed autologous conjunctival transplant (ACT) to treat injured cornea by ocular burn. In 1989, Kenyon and Tseng ${ }^{9}$ described autologous limbal transplant (ALT), where the contralateral corneoconjunctival limbus was included with the conjunctiva and transplanted to the deficient area, as being a good option for unilateral injury of the limbus. To treat severe bilateral injuries or in patients who do not allow surgical procedures, even minor, in the noncompromised eye, homologous limbal transplant (HLT) - that is, using cadaver donor tissue or tissue from a live related donor was suggested, but has the inconvenience of requiring immunosuppression. ${ }^{10-14}$ Amniotic membrane transplant (AMT) could be helpful in managing severe cases where subconjunctival fibrosis and chronic inflammation damage the basal membrane. ${ }^{6}$

We propose an alternative procedure, ipsilateral limbal transplantation (ILT) for patients with partial limbal deficiency secondary to alkali ocular burn.

\section{Material and methods}

Five eyes of five patients with partial limbal deficiency $(50 \%$ or less) secondary to ocular alkali burn were selected. All patients were male, three were black and two, white. Age ranged from 18 to 50 years old.

Preoperative evaluation included best corrected visual acuity (BCVA), biomicroscopy, tonometry, funduscopy, and ultrasonography. All patients signed an informed consent after clear and thorough explanation of the procedure.

Surgical technique consisted of translocation of the healthy portion of the limbus from the same eye, partially damaged, to the compromised area. The surgical technique is as follows. 
Table 1 Preoperative and postoperative best corrected visual acuity after ipsilateral limbal translocation in patients with partial limbal deficiency secondary to ocular alkali burn

\begin{tabular}{lllllll}
\hline Patient & Age & Affected eye & Preoperative VA & Postoperative VA & Refraction & $\begin{array}{c}\text { Follow up } \\
\text { (months) }\end{array}$ \\
\hline 1 & 38 & LE & HM & $20 / 40$ & $-2.00-4.00 \times 110^{\circ}$ & 11 \\
2 & 50 & LE & HM & $20 / 60$ & $+1.00 \times 180^{\circ}$ & 10 \\
3 & 34 & LE & $20 / 80$ & $20 / 20$ & $-1.50+2.50 \times 90^{\circ}$ & 9 \\
4 & 18 & RE & CF 3m & $20 / 60$ & $+1.50 \times 90^{\circ}$ & 7 \\
5 & 19 & LE & 20/200 & $20 / 60$ & $+2.00 \times 90^{\circ}$ & 7 \\
\hline
\end{tabular}

$\mathrm{VA}=$ visual acuity $\mathrm{LE}=$ left eye $\mathrm{RE}=$ right eye $\mathrm{HM}=$ hand movement; $\mathrm{CF} 3 \mathrm{~m}=$ counting fingers at 3 metres.

PREPARATION OF RECEPTOR BED

Under general or topical anaesthesia, peritomy adjacent to the deficient area was done, followed by removal of the conjunctivalisation.

OBTAINING DONOR LIMBUS

The size of donor area removed from the healthy limbus in the opposite meridian of the damaged limbus, might vary, according to each case. The average size in our cases was at least $2.5 \times 5.0 \mathrm{~mm}$ with $0.5-1.0 \mathrm{~mm}$ depth.

TRANSLOCATION OF THE STEM CELLS

After obtaining the donor limbus, it was carefully transferred to the previously prepared
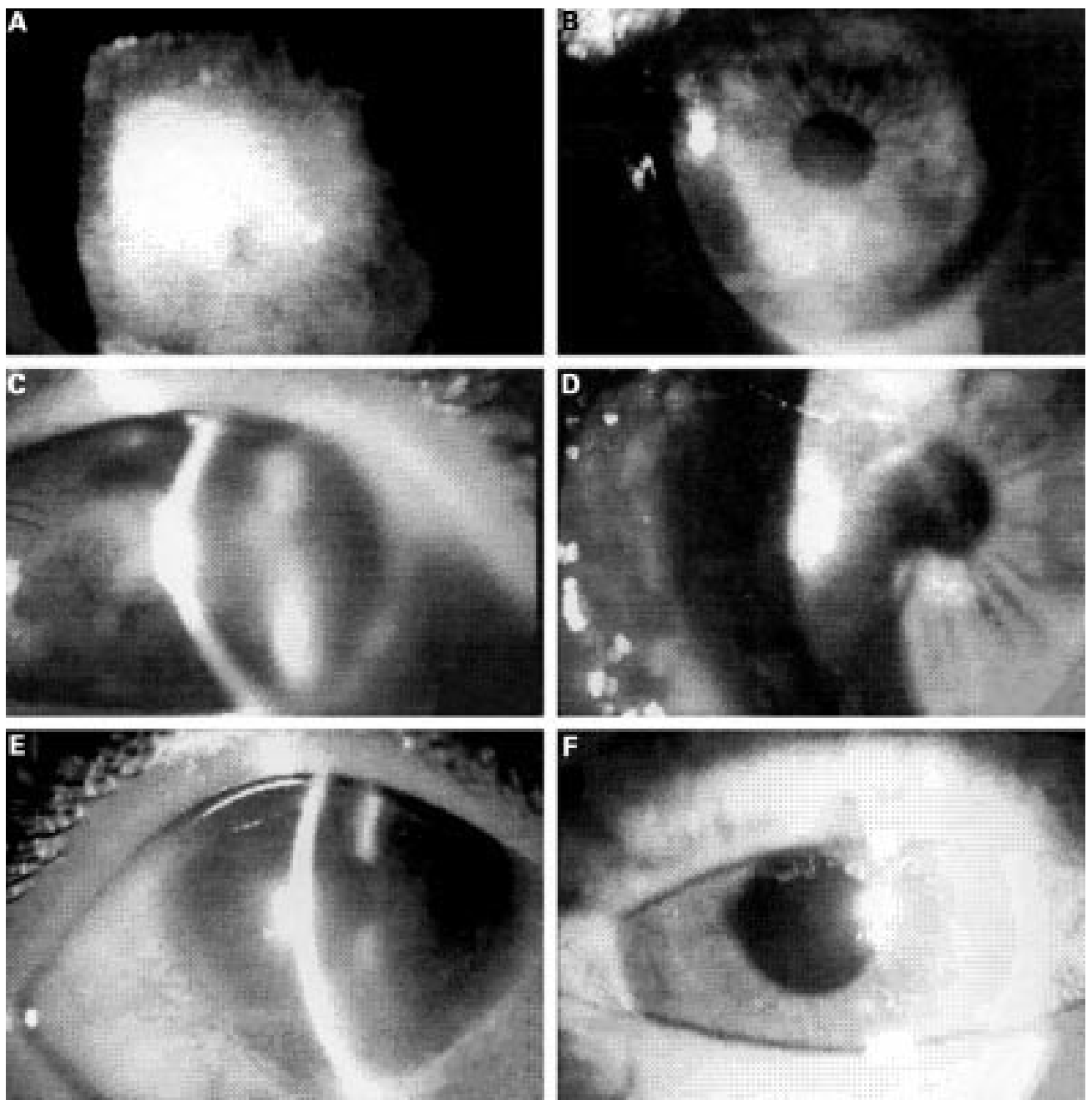

Figure 1 Preoperative and postoperative photographic sequence of three patients undergoing ILT. (A) Patient 1 with 4+ conjunctival hyperaemia, central epithelial defect, corneal oedema, neovascularisation, and BCVA of hand movements. (B) Same patient 11 months after ILT. Stable ocular surface and BCVA of 20/40. (C) Patient 2 presenting with diffuse conjunctival hyperaemia and corneal opacity with epithelial oedema, neovascularisation, and severe stromal oedema. BCVA was hand movements. (D) Same patient 10 months after ILT. BCVA was 20/60. (E) Patient 3 presenting epithelial defect, corneal oedema, and stromal opacity compromising the third inferior section of the cornea. BCVA was 20/80. (F) Nine months after ILT BCVA was 20/20.

receptor bed and fixed with interrupted 10-0 mononylon sutures, followed by subconjunctival injection of antibiotic (gentamicin) and steroids (dexamethasone), and therapeutic contact lens.

Postoperative medication consisted of topical antibiotics (ofloxacin) and steroids (1\% prednisolone acetate) every 6 hours for 2 weeks and then tapered. Patients were seen at days 1 , $7,15,30$, and then monthly, for at least 6 months. Suture removal was started after 30 days, but early removal was done in cases of loose suture, infiltrate, excessive discharge, or knot extrusion. Therapeutic contact lenses
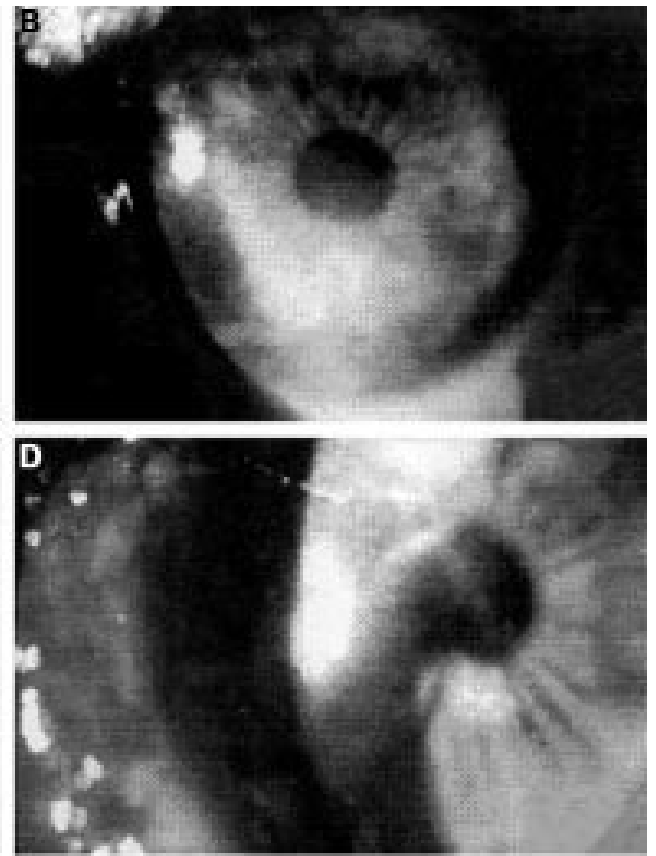
were removed after epithelial healing. BCVA, biomicroscopy at slit lamp, and tonometry were done at each follow up visit.

\section{Results}

The time interval between the ocular trauma and surgery ranged from 1 to 11 months (5.6 (SD 4.2) months). The follow up period ranged from 7 to 11 months

In all cases, the donor limbus was obtained from the superior limbus, intact and preserved in all cases, probably due to the superior eyelid protection, and transferred to the damaged area.

Postoperatively, completed corneal epithelialisation was seen in all cases after 1 month and did not recur. The fibrovascular tissue removed during surgery did not recur in any case. Corneal oedema resolved between 2 and 4 months after the procedure. Stromal corneal opacity and peripheral neovascularisation, present in all cases, regressed partially 1-4 months after surgery. BCVA improved in all cases and four patients presented with the rule astigmatism and one presented against the rule astigmatism (see Table 1 and Fig 1). None of our patients required a secondary penetrating keratoplasty (PKP) to improve vision.

\section{Discussion}

Previous studies have shown that ALT is an excellent treatment for eyes with limbal deficiency of different aetiologies. ${ }^{3910}$ ALT leads to fast recovery of ocular surface health, promotes progressive corneal epithelial healing, regression of corneal neovascularisation, and stromal inflammation. The BCVA improved in a large number of cases and when the PKP is required, the prognosis is better. ${ }^{3910}$

For bilateral injury, HTL was suggested as an option. However, there is a high risk of rejection and long term immunosuppression is fundamental in these cases. ${ }^{5} 101314$

All five cases with partial limbal deficiency secondary to chemical ocular burn reported here were successfully treated with ipsilateral autogenous limbal transplant. In all patients, there was complete re-epithelialisation, progressive regression of corneal oedema, control of stromal inflammation, and neovascularisation. BCVA improvement was achieved in 30 days, at most. There was no need for corneal transplant; besides, our first intention was to prepare the ocular surface for a secondary optical penetrating keratoplasty.

Chemical ocular burn usually damages the inferior portion of the cornea because the patients tend to close their eyes at the moment of the accident, preserving superior corneal and ocular surface of damage.
With the rule astigmatism was found in four of five patients and could be surgically induced, because in all procedures, the superior limbus was taken.

In conclusion, ILT is a therapeutic option for patients with partial limbus deficiency (compromising $50 \%$ or less of limbal circumference) secondary to chemical ocular trauma. In our opinion, ILT does not augment the stem cell population, but redistributes limbal cells to deficient areas, allowing uniform epithelial healing. This principle is similar to the approach suggested by Dua (personal communication, 1997) that scrapes away the conjunctival tissue growing over epithelial deficient area, allowing health limbus to produce epithelial cells to grow over the damaged area.

In summary, ILT has the following advantages:

- avoids intervention in the contralateral healthy eye;

- could be done in bilateral cases, if the compromised area does not go over $50 \%$ of limbal circumference, at least in one eye;

- does not require immunosuppression.

The authors do not have commercial interest in the products cited in this article.

1 Tseng SCG. Concept and applications of limbal stem cells. Eye 1989;3:141-57.

2 Kruse FE. Stem cells and corneal epithelium regeneration. Eye 1994;8:170-83.

3 Ronk JK, Esmenjaud SR, Osorio M, et al. Limbal conjunctival autograft in a subacute alkaline corneal burn. Cornea 1994;13:465-8.

4 Schermer A, Galvin S, Sun TT. Differentiation related expression of a major $64 \mathrm{~K}$ corneal keratin in vivo abnormal in culture suggests limbal location of corneal epithelial stem cells. F Cell Biol 1986;103:49-62.

5 Coster DJ, Aggarwal RK, Williams KA. Surgical management of ocular surface disorders using conjunctiva and stem cell allograft. Br f Ophthalmol 1995;79:977-82.

6 Kim JC, Tseng SCG. Transplantation of preserved human amniotic membrane for surface reconstruction in severely damaged rabbit corneas. Cornea 1995;14:473-84.

7 Brown SI, Bloomfield SE, Pierce DB. Follow up report on transplantation of the alkali burned cornea. Am f Ophthalmol 1974;77:538.

8 Thoft RA. Conjunctival transplantation. Arch Ophthalmol 1977;95:1425-7.

9 Kenyon KR, Tseng SCG. Limbal autograft transplantation for ocular surface disorders. Ophthalmology 1989;96:70923

10 Tan DTH, Fickers LA, Buckley RJ. Limbal transplantation. Ophthalmology 1996;103:29-36.

11 Chen JJY, Tseng SCG. Abnormal corneal epithelium wound healing in a partial thickness removal of limbal epithelium. Invest Ophthalmol Vis Sci 1991;32:2219-33.

12 Huang AJW, Tseng SCG. Corneal epithelial wound healing in the absence of limbal epithelium. Invest Ophthalmol Vis Sci 1991;32:96-105.

13 Tsai RJF, Tseng SCG. Human allograft limbal transplantation for corneal surface disorders reconstruction. Cornea 1994;13:389-400.

14 Tsubota K, Toda I, Saito H, et al. Reconstruction of the corneal epithelium by limbal allograft transplantation for severe ocular surface disorders. Ophthalmology 1995;102: 1486-96. 